\title{
Diels-Alder reactions of vinylboranes: a computational study on the boron substituent effects
}

\author{
María A. Silva, ${ }^{* a}$ Silvina C. Pellegrinet, ${ }^{*, b}$ and Jonathan M. Goodman*,a \\ ${ }^{a}$ Unilever Centre for Molecular Informatics. Department of Chemistry, University of Cambridge, \\ Lensfield Road, Cambridge, CB2 1EW, U.K. \\ ${ }^{b}$ Instituto de Química Orgánica y de Síntesis (CONICET) - Facultad de Ciencias Bioquímicas y \\ Farmacéuticas, U.N.R. Suipacha 531, Rosario (2000), Argentina \\ E-mail: mas65@hermes.cam.ac.uk,spellegr@fbioyf.unr.edu.ar,J.M.Goodman@ch.cam.ac.uk
}

\section{Dedicated to Professor Edmundo A. Rúveda on the occasion of his $70^{\text {th }}$ birthday and to Professor Roberto A. Rossi on occasion of his $60^{\text {th }}$ birthday}

(received 05 Sep 03; accepted 24 Nov 03; published on the web 09 Dec 03)

\begin{abstract}
The effect of substitution on boron in the Diels-Alder reactions of vinylboranes has been investigated by a computational study performed with the B3LYP/6-31G* method. Four vinylboranes with different electronic and steric properties were chosen to study the reactivity and the regio- and stereoselectivities patterns. In general, the calculations for the reactions with cyclopentadiene, trans-piperylene and isoprene are in good agreement with experimental results.
\end{abstract}

Keywords: Diels-Alder reactions, vinylboranes, substituent effect, computational study

\section{Introduction}

Continuing with our theoretical studies on the Diels-Alder reaction of boron-activated dienophiles, ${ }^{1,2}$ we have undertaken a further investigation to gain a deeper insight into the reactivity of these intriguing dienophiles. Singleton et al. have published the results of experimental studies on a series of substituted dialkylvinylboranes. ${ }^{3}$ Further studies from other laboratories introduced dihalovinylboranes as dienophiles for the [4+2] cycloaddition. ${ }^{4}$ The reactivity shown by vinylboranes and the ability to control the selectivity of the reaction by the choice of the substituents at the boron atom represent the most interesting features of these DielsAlder reactions.

Our previous results indicated that both endo and exo transition structures for the reactions of dimethylvinylborane and vinyl-9-BBN adopt classical [4+2] character. Nevertheless, we found that non-classical [4+3] secondary orbital interactions (SOIs) between the B and C1 accounted 
for the observed endo-selectivity, while the regioselectivity was explained either in terms of electronic or steric effects depending on the substitution pattern on the boron atom. While electronic effects appeared to control the regiochemistry of the reactions of unhindered vinylboranes, steric effects seem to dominate the course of the reactions for bulky systems such as vinyl-9-BBN.

To evaluate the effect of boron substitution on the reactivity and the selectivity of vinylboranes we have performed a theoretical study on the Diels-Alder reactions of four different vinylboranes (1-4). We intended to rationalise the experimental regioselectivities and stereoselectivities by computationally investigating the reactions of vinylboranes 1-4 with cyclopentadiene (5), trans-piperylene (6) and isoprene (7). These reactions were chosen by Singleton to tune the properties of vinylboranes. ${ }^{3 \mathrm{~b}}$ This theoretical study would, in turn, facilitate the rational design of new vinylboranes that could be used in novel transformations. The electron withdrawing effect of chlorine in $\mathbf{3}$ might increase the asynchronicity of the reaction. We also envisaged that the possible delocalisation in $\mathbf{4}$ would have an effect on the reactivity and the selectivity but the direction of this is hard to predict. We anticipated that the endo:exo selectivity would be low for cyclopentadiene (5).

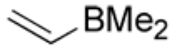

1

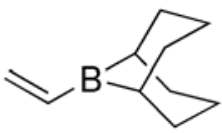

2

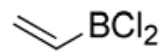

3

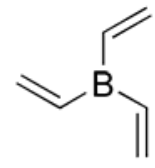

4

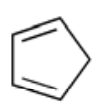

5

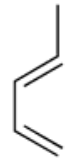

6

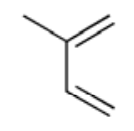

7

\section{Computational Methods}

Geometry optimizations of the transition structures and the reactants were carried out using the $\mathrm{B} \mathrm{LYP} \mathrm{P}^{5}$ functional with the $6-31 \mathrm{G}^{*}$ basis set. $^{6}$ Frequency calculations were used to confirm the nature of the stationary points. All transition state structures had only one imaginary frequency corresponding to the formation of the C-C bonds. Zero-point energies (ZPE) were computed at the B3LYP/6-31G* level without scaling. Natural bond orders (NBO) were calculated at the B3LYP/6-31G* level of theory. Optimizations and frequency calculations were carried out using GAMESS version 6/20/2002. ${ }^{7}$ NBO Analysis was preformed using the NBO 4.M program ${ }^{8}$ interfaced to the Jaguar 4.2 program. ${ }^{9}$ 


\section{Results and Discussion}

\section{Reactions with cyclopentadiene}

For the reaction with cyclopentadiene (5), there are two different transition structures that lead to the endo and exo products (Scheme 1).

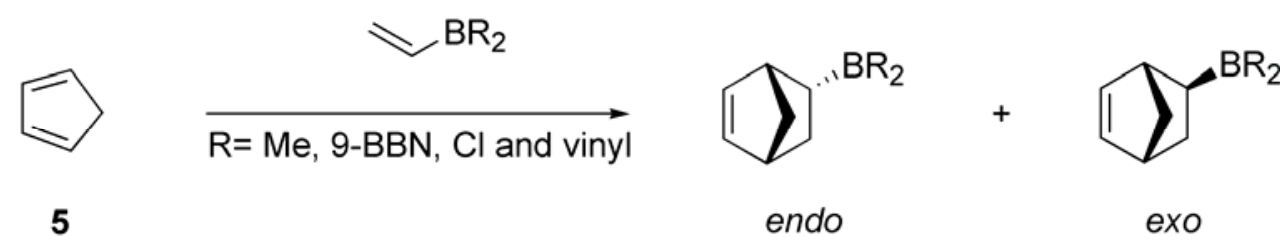

\section{Scheme 1}

In accordance with our earlier studies, ${ }^{1}$ we have found that the optimized geometries for the transition structures exhibit large degrees of asynchronicity (Figure 1). The geometrical features of these transition structures are also similar to the ones previously reported, with much shorter distances for C2-C3 than for C1-C6. In addition, C-B distances adopt shorter values for endo TS than the exo counterparts. ${ }^{10}$

Table 1 compares the calculated ratios ${ }^{11}$ of all the systems analyzed in this study with the experimental results ${ }^{12}$. Table 2 collects the energy data associated with the reaction with cyclopentadiene (5). In general, calculations reproduced the experimental endo:exo ratios acceptably, although endo-selectivities with cyclopentadiene were underestimated for most systems. For vinyl-9-BBN (2), theoretical results predict low endo:exo stereoselectivity that has also been found experimentally. The difference between the 2:1 ratio found experimentally and the calculated 1:2 ratio is a consequence of a small energy difference, and results on a correct prediction of low selectivity. In addition, for trivinylborane (4), calculations nicely match the experimental results (Figure 1). The reaction with cyclopentadiene (5) for all the dienophiles studied resulted in moderate to low endo-selectivities compared to the high endo-selectivities observed in the case of trans-piperylene (6). The endo-selectivities can be explained in terms of the [4+3] C-B secondary orbital interactions being slightly stronger in the case of the endo transition structures. NBO analysis agrees with this observation since C-B bond orders were always higher for the endo transition structures (0.10-0.07 for endo TS and 0.07-0.05 for exo TS). Close contacts between the atoms of the ligands on the boron and the hydrogens of the cyclopentadiene (5) seem to oppose electronic effects by destabilizing the endo TS relative to its exo counterpart for the reaction of the bulky dienophile 2. 


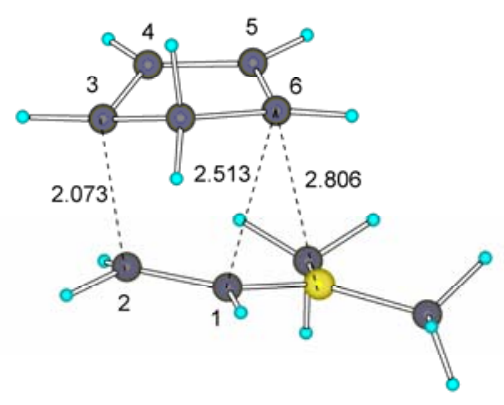

1AN

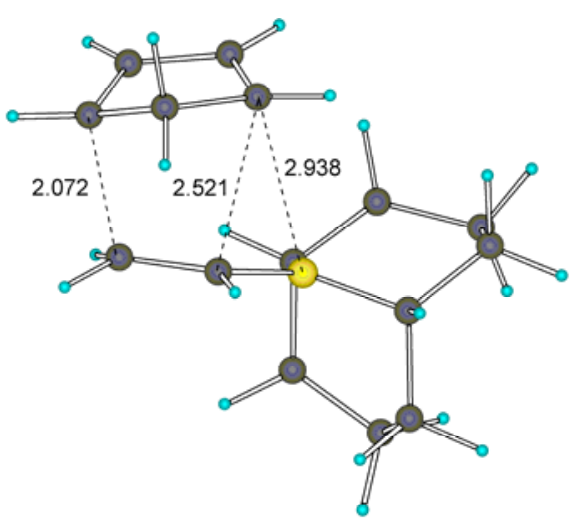

2AN
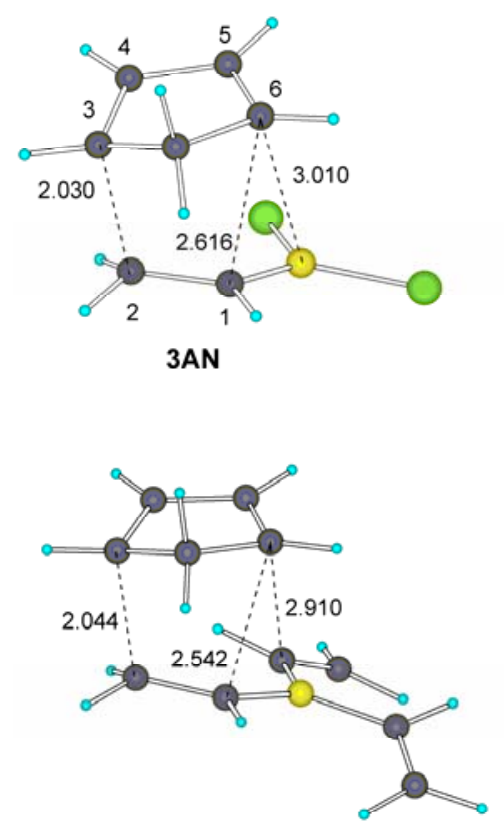

4AN
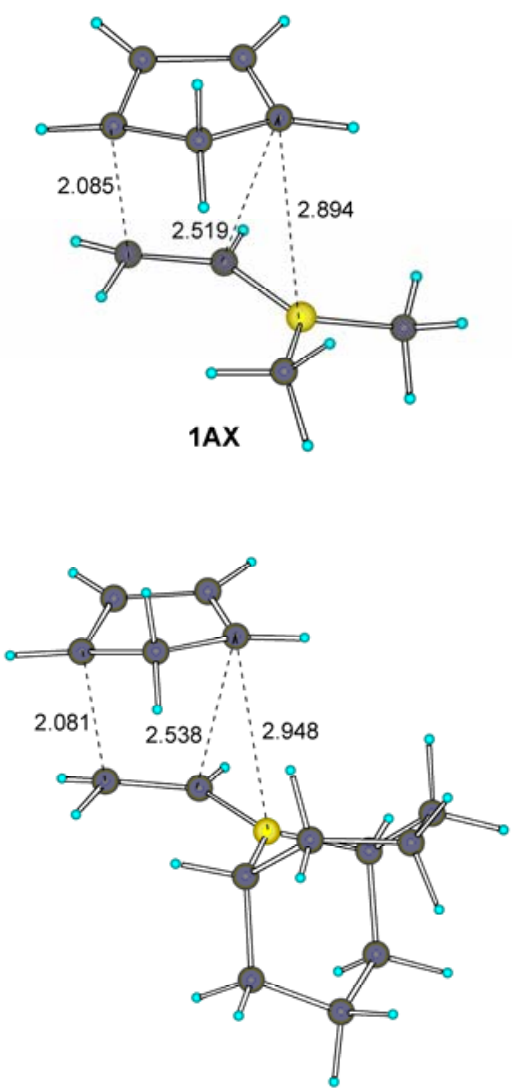

2AX
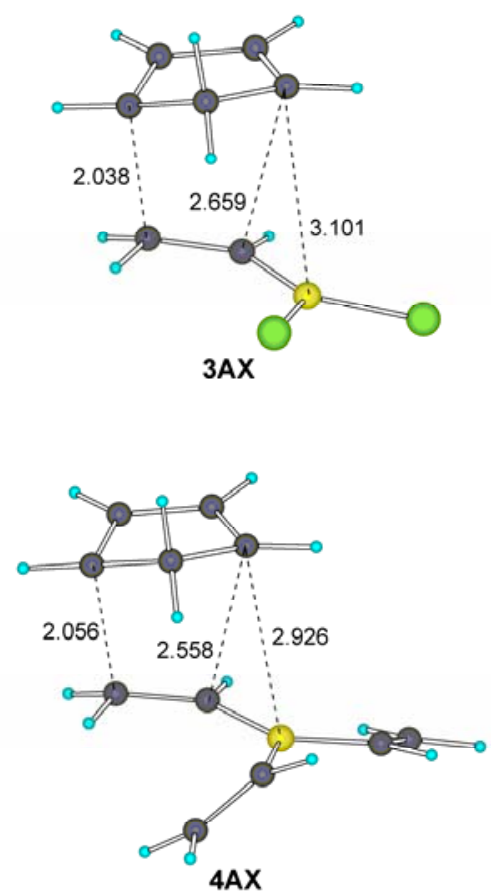

Figure 1. Transition structures for the reactions of vinylboranes 1-4 with cyclopentadiene (5) with B3LYP/6-31G*. All distances in A. 
Table 1. Experimental and calculated endo-stereoselectivity and regioselectivity of the DielsAlder reactions between vinylboranes 1-4 and dienes 5-7

\begin{tabular}{|c|c|c|c|c|c|c|c|c|}
\hline \multirow{2}{*}{$\begin{array}{c}\text { endo-exo } \\
\text { Stereoselectivity }\end{array}$} & \multicolumn{2}{|c|}{1} & \multicolumn{2}{|c|}{2} & \multicolumn{2}{|c|}{3} & \multicolumn{2}{|c|}{4} \\
\hline & Exp. & Calcd. & Exp. & Calcd. & Exp. & Calcd. & Exp. & Calcd. \\
\hline 5 & $84: 16$ & $67: 33$ & $67: 33$ & $31: 69$ & $84: 16$ & $60: 40$ & $78: 22$ & $85: 15$ \\
\hline 6 & $90: 10$ & $93: 7$ & $92: 8$ & $97: 3$ & - & $83: 17$ & $90: 10$ & $91: 9$ \\
\hline \multirow{2}{*}{ Regioselectivity } & \multicolumn{2}{|c|}{1} & \multicolumn{2}{|c|}{2} & \multicolumn{2}{|c|}{3} & \multicolumn{2}{|c|}{4} \\
\hline & Exp. & Calcd. & Exp. & Calcd. & Exp. & Calcd. & Exp. & Calcd. \\
\hline 6 & $50: 50$ & $56: 44$ & $2: 98$ & $10: 90$ & - & $97: 3$ & $49: 51$ & $89: 11$ \\
\hline 7 & $61: 39$ & $73: 27$ & $93: 7$ & $93: 7$ & $60: 40$ & $79: 21$ & $62: 38$ & $64: 36$ \\
\hline
\end{tabular}

${ }^{\text {a }}$ Ratios calculated including ZPE.

${ }^{\mathrm{b}}$ The ratios given are for ortho:meta products for trans-piperylene (6) and for para:meta products for isoprene (7).

Table 2. B3LYP/6-31G* Energies Relative to the Reactants (kcal/mol) Including ZPE Corrections for the Transition Structures of the Diels-Alder Reactions of Vinylboranes 1-4 with Cyclopentadiene (5). Relative Energies (kcal/mol) in parentheses

\begin{tabular}{cccc}
\hline TS & $\Delta E_{0}^{\ddagger}$ & TS & $\Delta E_{0}^{\ddagger}$ \\
\hline $\mathbf{1 A N}$ & $17.67(0.00)$ & 3AN & $14.90(0.00)$ \\
$\mathbf{1 A X}$ & $18.09(0.42)$ & 3AX & $15.14(0.23)$ \\
& & & \\
$\mathbf{2 A N}$ & $18.78(0.48)$ & $\mathbf{4 A N}$ & $18.22(0.00)$ \\
$\mathbf{2 A X}$ & $18.30(0.00)$ & $\mathbf{4 A X}$ & $19.27(1.05)$ \\
\hline
\end{tabular}

In most cases, the energy barriers collected in Table 2 are in accordance with the reactivity trends. Experiments demonstrated that trivinylborane is more reactive towards cyclopentadiene (5) than both dimethylvinylborane (1) and vinyl-9-BBN (2). ${ }^{3 b}$ In addition, dichlorovinylborane has been shown to react faster with 2,3-dimethyl-1,3-butadiene than dialkylvinylboranes. ${ }^{4 b}$ Calculations correctly reproduced that dichlorovinylborane (3) is the most reactive dienophile due the presence of electron-withdrawing chlorine atoms on the boron atom and vinyl-9-BBN (2) is the least reactive because of its important steric hindrance. The results of the calculations of trivinylborane (4) do not fit into the reactivity trend.

The effect of the structure of the dienophile on the regioselectivity of these reactions was considered by the use of trans-piperylene (6) and isoprene (7) (Schemes 2 and 3). 


\section{Reactions with trans-piperylene}

In the case of trans-piperylene (6), there are four transition structures (endo/exo and ortho/meta, Scheme 2, Table 3).

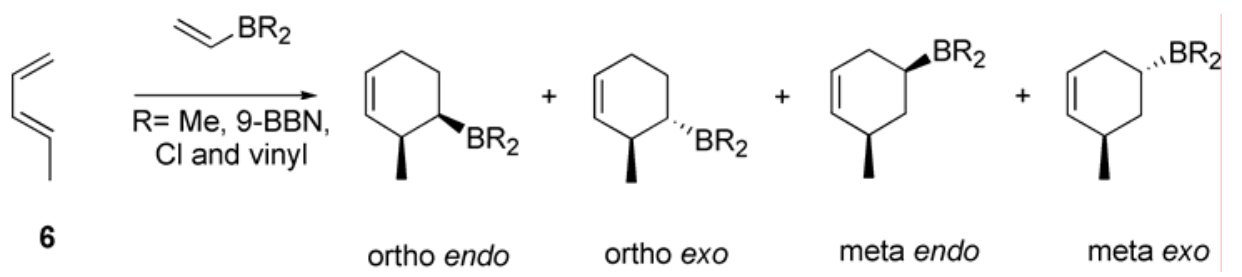

\section{Scheme 2}

Table 3. B3LYP/6-31G* Energies Relative to the Reactants $(\mathrm{kcal} / \mathrm{mol})$ Including ZPE Corrections for the Transition Structures of the Diels-Alder Reactions of Vinylboranes 1-4 with Trans-piperylene (6). Relative Energies (kcal/mol) in parentheses

\begin{tabular}{cccc}
\hline TS & $\Delta E_{0}^{\ddagger}$ & TS & $\Delta E_{0}^{\ddagger}$ \\
\hline 1BON & $20.16(0.00)$ & 3BON & $16.73(0.00)$ \\
1BOX & $21.72(1.56)$ & 3BOX & $17.44(0.70)$ \\
1BMN & $20.34(0.18)$ & 3BMN & $18.85(2.12)$ \\
$\mathbf{1 B M X}$ & $21.53(1.38)$ & 3BMX & $19.80(3.07)$ \\
& & & \\
2BON & $21.93(1.39)$ & 4BON & $19.53(0.00)$ \\
2BOX & $23.00(2.46)$ & $\mathbf{4 B O X}$ & $20.94(1.41)$ \\
$\mathbf{2 B M N}$ & $20.54(0.00)$ & $\mathbf{4 B M N}$ & $20.74(1.21)$ \\
$\mathbf{2 B M X}$ & $22.68(2.14)$ & $\mathbf{4 B M X}$ & $22.42(2.89)$ \\
\hline
\end{tabular}

The calculations performed with trans-piperylene (6) are in good agreement with the experimental results for $\mathbf{1}$ and 2. The endo-stereoselectivies of these reactions are correctly computed to increase compared to those corresponding to the reactions with cyclopentadiene (5). NBOs indicate that when trans-piperylene is used as the diene, C-B [4+3] SOIs increase the relative stabilization of endo TS compared to the exo TS. The smaller difference between NBOs of endo and exo TS for the reactions of cyclopentadiene (5) could be a consequence of the steric attenuation of SOI. The lack of regioselectivity for the reaction with dimethylvinylborane (1) was predicted and the preferred anomalous meta orientation with vinyl-9-BBN (2) was also reproduced, albeit underestimated. To our surprise, a very high ortho regioselectivity was calculated in the case of dichlorovinylborane (3), with a very asynchronous transition structures, showing a difference between C1-C6 and C2-C3 distance around $0.9 \AA$. The special stabilization observed for the ortho-endo transition structure with trans-piperylene (6) (3BON) clearly 
contrasts with its weaker C-B interaction compared to the one corresponding to the meta-endo transition structure (3BMN), as shown by the values of the C-B bond orders being lower for the former (bond order values for 3BON and 3BMN are 0.07 and 0.11 , respectively). However, the geometry of 3BON shows the proximity of one of the protons in the methyl group of the diene with one of the chlorine atoms of the dienophile and demonstrates the presence of a stabilizing interaction (Figure 2). This interaction is supported by a weak NBO value of 0.01 between the hydrogen and the chlorine atoms. This kind of $\mathrm{H}$-halide interaction has been previously observed for carbonyl-boron Lewis acid complexes. ${ }^{13}$

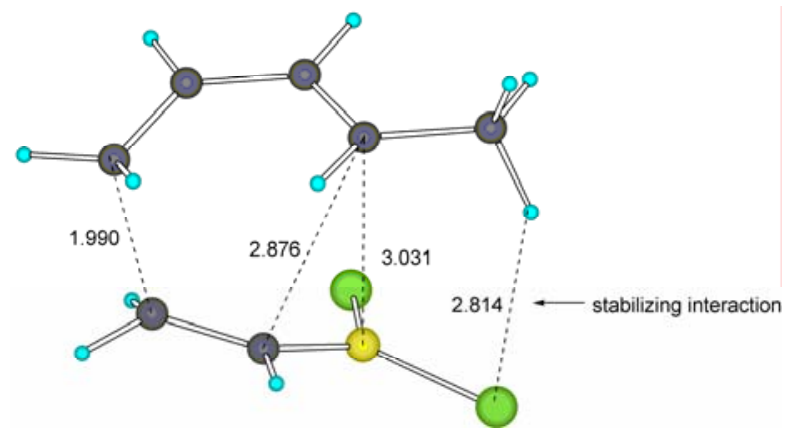

Figure 2. Hydrogen-chlorine interaction in the transition structure 3BON.

The disagreement found between the calculation and the experimental result obtained for the reaction of trivinylborane (4) with trans-piperylene (6) was surprising and we decided to investigate this system further. The flexibility showed by trivinylborane (4) was the first issue that concerned us. The transition structures initially found had trivinylborane (4) almost planar. We decided to search for different conformations of the vinyl groups in an attempt to find more stable transition structures that could reproduce the regiochemical outcome for this reaction. ${ }^{14}$ Although we indeed found different genuine transition structures that differed in the conformation of the vinyl groups, we could not find any transition structure significantly lower in energy. Nevertheless, we calculated the ratios taking all the located TS into account, and the ratio slightly changed towards the experimental ratio. We are pursuing further studies on this system.

\section{Reactions with isoprene}

The Diels-Alder reactions with isoprene (7) lead to two regioisomeric products through four different transition structures, endo/exo and para/meta (Scheme 3, Table 4). Each product can be formed from both the endo and exo approaches corresponding to the same regiochemistry. 


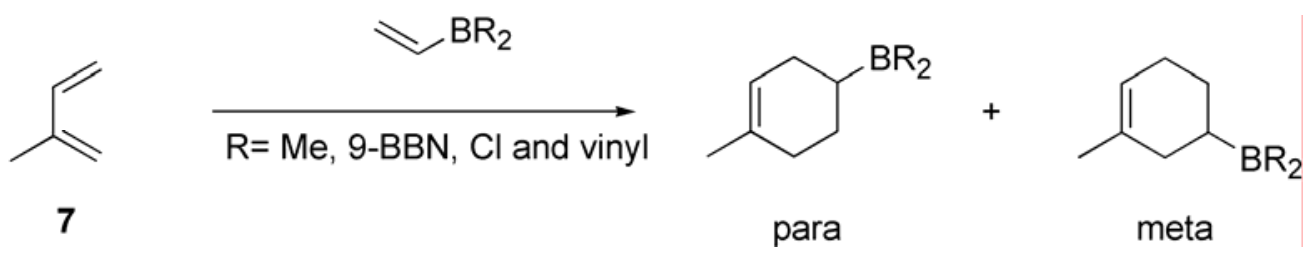

\section{Scheme 3}

Table 4. B3LYP/6-31G* Energies Relative to the Reactants $(\mathrm{kcal} / \mathrm{mol})$ Including ZPE Corrections for the Transition Structures of the Diels-Alder Reactions of Vinylboranes 1-4 with Isoprene (7). Relative Energies (kcal/mol) in parentheses

\begin{tabular}{cccc}
\hline TS & $\Delta E^{\ddagger}$ & TS & $\Delta E_{0}^{\ddagger}$ \\
\hline $\mathbf{1 C P N}$ & $17.84(0.00)$ & $\mathbf{3 C P N}$ & $15.97(0.00)$ \\
$\mathbf{1 C P X}$ & $19.49(1.65)$ & $\mathbf{3 C P X}$ & $17.15(1.17)$ \\
$\mathbf{1 C M N}$ & $18.43(0.58)$ & $\mathbf{3 C M N}$ & $16.75(0.78)$ \\
$\mathbf{1 C M X}$ & $20.62(2.77)$ & $\mathbf{3 C M X}$ & $18.20(2.23)$ \\
& & & \\
$\mathbf{2 C P N}$ & $18.60(0.00)$ & $\mathbf{4 C P N}$ & $18.38(0.00)$ \\
$\mathbf{2 C P X}$ & $20.50(1.90)$ & $\mathbf{4 C P X}$ & $20.44(2.06)$ \\
$\mathbf{2 C M N}$ & $20.24(1.64)$ & $\mathbf{4 C M N}$ & $18.71(0.33)$ \\
$\mathbf{2 C M X}$ & $21.40(2.81)$ & $\mathbf{4 C M X}$ & $21.18(2.80)$ \\
\hline
\end{tabular}

Dimethylvinylborane (1), dichlorovinylborane (3) and trivinylborane (4) exhibit similar degrees of regioselectivities in the reactions with isoprene (7) and the calculated regioselectivities are in excellent agreement with the experimental results. The high para regioselectivity observed in the case of vinyl-9-BBN (2) is correctly predicted. Analysis of the NBOs and the close contacts in the transition structures of these reactions suggests that the regiochemical outcome is finely balanced between electronic and steric effects and the high para regioselectivity observed in the case of vinyl-9-BBN (2) is due to unfavorable steric interactions in the meta transition structures.

\section{Conclusions}

The substituent effect on the boron atom of vinylboranes in the Diels-Alder reaction with different dienes is acceptably well reproduced using DFT methods at the B3LYP level. Calculations are in accordance with the fact that dimethylvinylborane, dichloroborane and trivinylborane exhibit very similar regio- and stereoselectivities with the dienes under study. Thus, this investigation corroborates the modest relevance of electronic effects on boron. However, steric effects seem to play a decisive role since the bulky vinyl-9-BBN displays 
enhanced regioselectivity and lower endo-selectivity with cyclopentadiene. The most significant discrepancy between experimental results and calculations is in the regioselectivity for the reaction of trivinylborane (4) and trans-piperylene (6). This reveals a possible anomalous behavior of $\mathbf{4}$ due to its greater reactivity, and so a greater possibility that the reaction is reversible. Furthermore, dichlorovinylborane (3) showed high ortho regioselectivity with transpiperylene (6), provided by an interesting stabilizing $\mathrm{H}-\mathrm{Cl}$ interaction.

The present investigation extended previous computational studies on the Diels-Alder reactions of boron-activated dienophiles and should contribute to promote further research on this area.

\section{Acknowledgments}

We thank CONICET, Universidad Nacional de Rosario and Fundación Antorchas for financial support. M.A.S. thanks the European Community for a Marie Curie fellowship. We thank the UK computational chemistry facility (UKCCF) for computer resources.

\section{Supplementary Information Available}

Selected distances (in $\AA$ ) and bond orders for all the transition structures studied in the paper (Table S1 - page 584). Thermochemical Data $(\mathrm{kcal} / \mathrm{mol})$ for all the transition structures studied in the paper (Table S2 - page 586). Cartesian coordinates and absolute energies (in hartrees), including ZPE, and number of imaginary frequencies of all stationary points reported in the paper; values of imaginary frequencies of all transition structures. B3LYP/6-31G* optimized geometries for the transition structures not shown in the paper (Figures S1 - S4 - page 609).

\section{References and Notes}

1. Pellegrinet, S. C.; Silva, M. A.; Goodman, J. M. J. Am. Chem. Soc. 2001, 123, 8832.

2. (a) Silva, M. A.; Pellegrinet, S. C.; Goodman, J. M. J. Org. Chem. 2002, 67, 8203. (b) Silva, M. A.; Pellegrinet, S. C.; Goodman, J. M. J. Org. Chem. 2003, 68, 4059.

3. (a) Singleton, D. A.; Martinez, J. P. J. Am. Chem. Soc. 1990, 112, 7423. (b) Singleton, D. A.; Martinez, J. P.; Watson, J. V.; Ndip, G. M. Tetrahedron 1992, 48, 5831. (c) Singleton, D. A.; Martinez, J. P.; Ndip, G. M. J. Org. Chem. 1992, 57, 5768. (d) Singleton, D. A.; Kim, K.; Martinez, J. P., Tetrahedron Lett. 1993, 34, 3071.

4. (a) Noiret, N.; Youssofi, A.; Carboni, B.; Vautier, M. J. Chem. Soc., Chem. Commun. 1992, 1105. (b) Zaidlewicz, M.; Binkul, J.R.; Sokól, W. J. Organomet. Chem. 1999, 580, 354. 
5. (a) Becke, A. D. J. Chem. Phys. 1993, 98, 5648. (b) Lee, C.; Yang, W.; Parr, R. Phys. Rev. B 1988, 37, 785. (c) Hehre, W. J.; Radom, L.; Schleyer, P. v. R.; Pople, J. A. Ab Initio Molecular Orbital Theory; Wiley: New York, 1986.

6. Hehre, W. J.; Radom, L.; Schleyer, P. v. R.; Pople, J. A. Ab Initio Molecular Orbital Theory; Wiley: New York, 1986.

7. Schmidt, M. W.; Baldridge, K. K.; Boatz, J. A.; Elbert, S. T.; Gordon, M. S.; Jensen, J. H.; Koseki, S.; Matsunaga, N.; Nguyen, K. A.; Su, S. J.; Windus, T. L.; Dupuis, M.; Montgomery, J. A. J. Comput. Chem. 1993, 14, 1347.

8. Glendening, E. D.; Badelhoop, J. K.; Reed, A. E.; Carpenter, J. E.; Weinhold, F. NBO 4.M; Theoretical Chemistry Institute: University of Wisconsin, Madison, 1999.

9. Jaguar 4.2, Schrodinger, Inc., Portland, Oregon, 2000.

10. We named the transition states as follows: the number indicates the dienophile, i.e. 1 for dimethylvinylborane (1), 2 for vinyl-9-BBN (2), 3 for dichlorovinylborane (3) and 4 for trivinylborane (4). Then we added A for cyclopentadiene (5), B for (E)-1,3-pentadiene (trans-piperylene, 6) and C for 2-methylbutadiene (isoprene, 7). Finally, we used O, M and P for ortho, meta and para regiochemistry and $\mathrm{N}$ and $\mathrm{X}$ for endo and exo stereochemistry, respectively. For example, $\mathbf{3 B M X}$ is the transition structure corresponding to the reaction between dichlorovinylborane and trans-piperylene leading to the meta regioisomer with an exo stereochemistry.

11. All product ratios were derived from the Boltzmann distributions computed from the equation: $\mathrm{k}_{1} / \mathrm{k}_{2}=\mathrm{e}^{-\Delta \mathrm{E} \neq / \mathrm{RT}}$, where $\Delta \mathrm{E}^{\ddagger}$ is the difference between the calculated activation energies, taken from Tables 2-4, for any two processes, $\mathrm{T}=298.15 \mathrm{~K}$ and $\mathrm{R}=1.9872 \mathrm{cal} \mathrm{K}^{-1}$ $\mathrm{mol}^{-1}$. We also performed the thermochemical analysis for the reactions under study and calculated the relative free energies. These values were very similar to those corresponding to the zero-point corrected energies and led to comparable calculated ratios. This result suggests entropic effects in isomeric transition structures are uniform for these reactions, which is in accord with previous results.

12. Experimental ratios were taken from Refs. 3 and 4. For the reactions of vinyldimethylborane and trivinylborane with isoprene, we used the data obtained experimentally for myrcene, a diene structurally similar to isoprene.

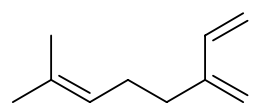

myrcene

13. Mackey, M. D.; Goodman, J. M. Chem. Comm. 1997, 2383.

14. The two conformations $\mathbf{4 a}$ and $\mathbf{4 b}$ for trivinylborane (4) were studied and no significant difference in energy $(0.3 \mathrm{kcal} / \mathrm{mol})$ was calculated. $\mathbf{4 a}$ is almost planar, $\mathbf{4 b}$ is less so.

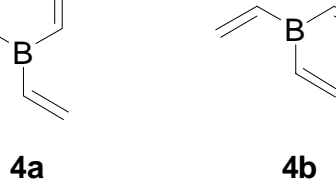

